\title{
A Superconducting Solenoid and Press for Permanent Magnet Fabrication
}

\author{
Thomas M. Mulcahy and John R. Hull
}

\begin{abstract}
For the first time, a superconducting solenoid (SCM) was used to increase the remnant magnetization of sintered NdFeB permanent magnets (PMs). In particular, improved magnetic alignment of commercial-grade $P M$ powder was achieved, as it was axial die pressed into $12.7-\mathrm{mm}$ diameter cylindrical compacts in the $76.2-\mathrm{mm}$ warm bore of a 9-T SCM. The press used to compact the powder is unique and was specifically designed for use with the SCM. Although the press was operated in the batch mode for this proof of concept study, its design is intended to enable automated production. In operation, a simple die and punch set made of nonmagnetic materials was filled with powder and loaded into a nonmagnetic press tube. The cantilevered press tube was inserted horizontally, on a carrier manually advanced along a track, into the SCM. The robustness of the mechanical components and the SCM, in its liquid helium dewar, were specifically designed to allow for insertion and extraction of the magnetic powder and compacts, while operating at $9 \mathrm{~T}$. Compaction was achieved by pressing the punches between the closed end of the press tube and the hydraulic cylinder mounted on the opposite end. Improvements up to $10 \%$ in magnetization and $20 \%$ in energy products of the permanent magnets were obtained, as the alignment fields were increased above the 2-T maximum field of the electromagnets used in industry. Increases in magnetization of $3 \%$ are significant in the mature sintered magnet industry.
\end{abstract}

Index Terms - Anisotropic permanent magnet fabrication, low-temperature superconducting solenoid, magnet powder alignment and compaction.

\section{INTRODUCTION}

$\mathrm{T}$ he cost, weight, and volume of existing electric traction motors is too large to meet the performance targets established for advanced vehicles. One of the most promising areas to reduce weight and volume in a motor is by increasing the energy product of the permanent magnets (PMs) used in the motors. Higher energy products will not only reduce the weight of the PM, but will also reduce by a similar fraction the weight in the balance of a motor producing the same torque. Weight reductions to $20 \%$, and associated lower costs, are anticipated, if high-field superconducting solenoids (SCMs) are used in the processing of permanent magnets instead of industry's 2-T electromagnets. Experimental proof of the concept is presented here.

Manuscript received August 4, 2002. The U.S. Department of Energy, Offices of Advanced Automotive Technologies, supported the work under Contract W-31-109-Eng-38.

Authors are with Argonne National Laboratory, Argonne, IL 60439 (telephones: 630-252-6145 and 630-252-8580, e-mails: mulcahy@anl.gov and jhull@anl.gov).

\section{A. Why use SCMs in Sintered Anisotropic PM Fabrication?}

An important step in the fabrication of the highest energy product PMs is the unidirectional magnetic alignment of the $3-\mu$ to $5-\mu$ single-crystal grains, while the powder is pressed into compacts for sintering [1]. The PM's remnant magnetism is proportional to $\cos (\theta)$, where $\theta$ is the angle between each grain's easy magnetic axis and the desired direction of alignment. For perfect alignment $\theta=0$ for each grain, but this is never realized. Primarily, grain alignment is limited by mechanical friction between grains and the selffields generated by the powder volume in the die.

Today's permanent magnet producers use $1-\mathrm{T}$ to $2-\mathrm{T}$ pulsed electromagnets in axial die pressing, to align the grains in the press direction. Axial die pressing dominates anisotropic magnet production because it is the most cost effective and best suited for making near-final-shape magnets - a current goal of industry. But alignment is far from complete, even when organic lubricants are added to the magnet powder to promote rotation of the grains. In particular, the use of lubricants is limited because they oxidize the free $\mathrm{Nd}$, which is present in the powder as a sintering aid and for producing magnetic coercivity. Adding more paramagnetic $\mathrm{Nd}$ is not a solution, because this reduces the percentage of hard magnetic material and, therefore, the remnant magnetization of the PM. Just as importantly, too much lubricant weakens the strength of the compact. Press loads are minimized to just hold the compacts together for handling. Larger loads increase the partial buckling of the columns of grains and their misalignment. Expectations are that the higher fields of SCMs, and the greater torque on each grain, will improve and maintain grain alignment before and during pressing, and, thus, produce better PMs.

The other major cause of grain misalignment is associated with the magnetization of finite size volumes of powder. When the magnetic powder in the die is subject to a uniform external alignment field, a nonuniform self-field occurs spontaneously. Since the magnetic axes of the grains align along the combined field lines, unidirectional alignment of the grains is distorted. Most unfortunately, the self-field and distortion is greatest for compacts with the smaller length to diameter ratios that are typical of near-final-shape magnets. However, the higher alignment fields of SCMs are expected to improve the alignment of near-final-final-shape magnets by overwhelming the self-field distortions. For example, field lines calculated [2] with the electromagnetic code Vectra are shown in Fig. 1 for alignment fields of 1-T and 8-T, applied to the same finite-length powder filled die cavity. Clearly, the potential exists for improved grain alignment using an SCM. 


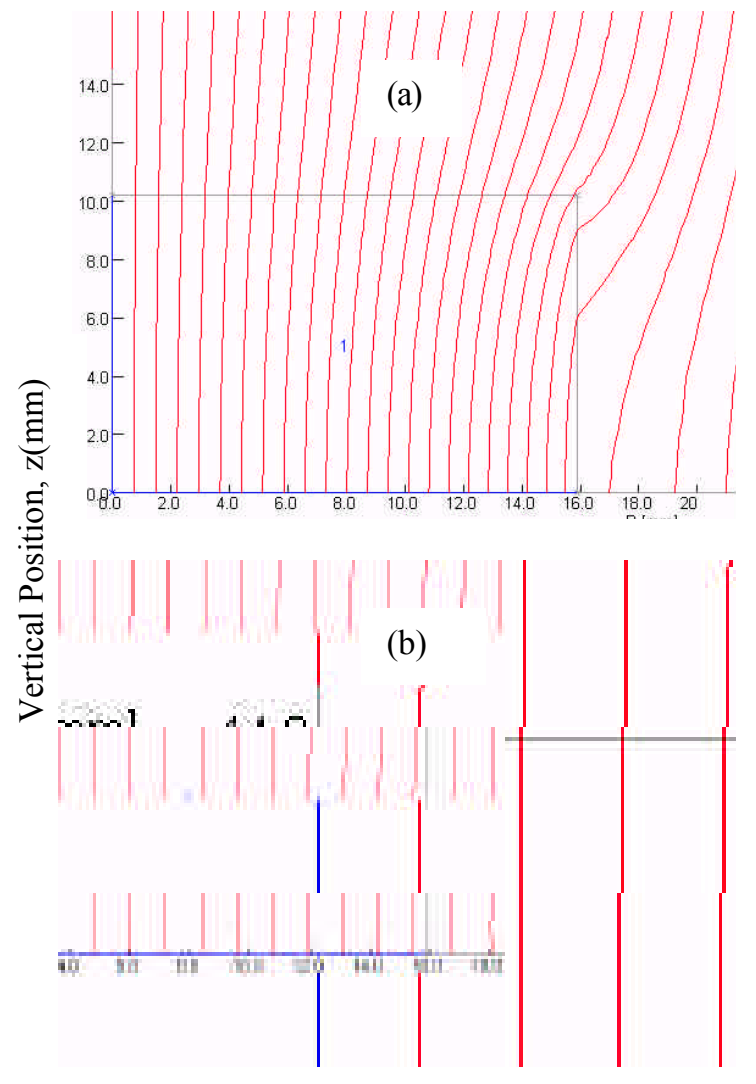

Radial Position, r(mm)

Fig. 1. Field lines for one-quarter of a cylindrical compact vertically pressed in an alignment field of (a) $\mathrm{H}=1 \mathrm{~T}$ and (b) $\mathrm{H}=8 \mathrm{~T}$. Compact's length/diameter, $\mathrm{L} / \mathrm{D}=0.64$.

\section{B. Industrial Presses}

The industry uses commercially available vertical hydraulic presses to compact the PM powder [1]. Typically, each magnet producer will add custom designed electromagnets that surround the upper and lower punches, as well as the die-cavity. The magnet designs take into account the ferromagnetic steel of the press and die, for each shape magnet produced. Usually the designs are done in-house and, at least, are proprietary, because their design significantly affects product quality.

Industry's electromagnets are turned on only while the powder is being compacted. Thus, the die can be filled with magnet powder, and the compact extracted from the die, inside and near the inactive electromagnet. Typical manual rates of compact production are one every couple of minutes. When operations are completely automated, several compacts may be produced per minute. Since SCMs can not be cycled on and off that fast, the SCM and press design must accommodate operations in a constant and high DC field.

\section{TEST FACILITY}

The purpose of the facility is to demonstrate that the magnetic properties of axial die-pressed PMs can be significantly improved by pressing their compacts in the higher alignment fields available with an SCM. Higher fields are expected to increase the grain alignment of the compacts and, thus, the magnetic properties of the PMs. Improvements are assessed by comparing the residual magnetic flux densities and energy products of PMs that are compacted in alignment fields which vary from 1-T to 8-T. For this proof-of-concept study, the facility is manually operated, however operations critical for automation also are proven.

\section{A Reciprocating Press Concept}

To eventually achieve automated operation, the design concept chosen was to reciprocate the press into and out of the active SCM. A similar concept has already been used with superconducting solenoids in the clay-separation industry [3]. An unorthodox press-in-tube method for axialdie pressing in a batch (manual) mode was designed and built by Ability Engineering Technology, Inc., which met the magnetic and geometric constraints created by the SCM. See Fig. 2.

In operation, a simple die and punch set made of nonmagnetic materials is filled with powder in a nitrogen atmosphere glovebox and then loaded into the nonmagnetic press tube. Next, the cantilevered press tube is inserted horizontally into the warm bore, on an aluminum carrier that is manually advanced along an aluminum track. Precise centering of the press tube in the bore is maintained. Compaction is achieved by pressing the punches between the base plug at the end of the press tube and the hydraulic cylinder mounted on the opposite end. Finally, the press tube is extracted from the bore and the die containing the compact is removed from press tube and transferred to a glovebox, where a hand press is used to eject the compact.

The press tube and ram are made from very low relative magnetic permeability materials $(<1.002)$. The hydraulic cylinder was custom made, by Atlas Cylinders, Inc., from stainless steel with a low relative permeability $(<1.01)$. Electromagnetic code calculations were made to determine the

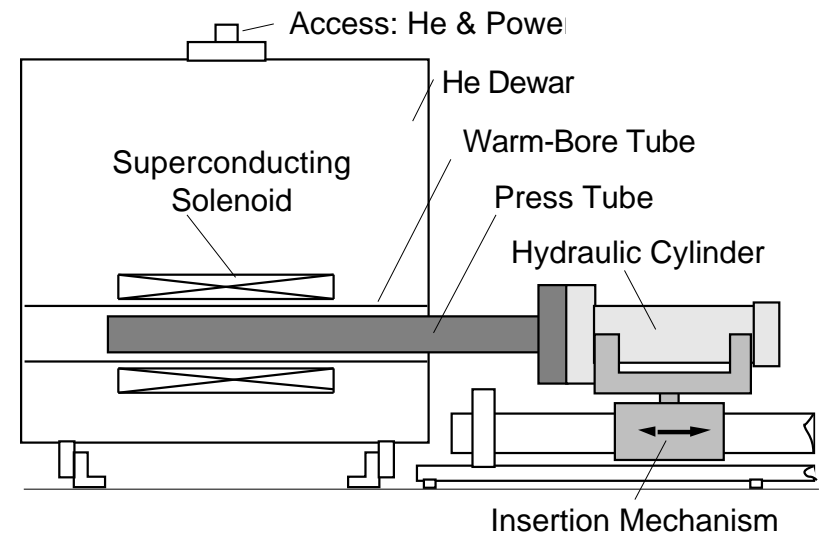

Fig. 2(a). Axial-die press tube in the SCM.

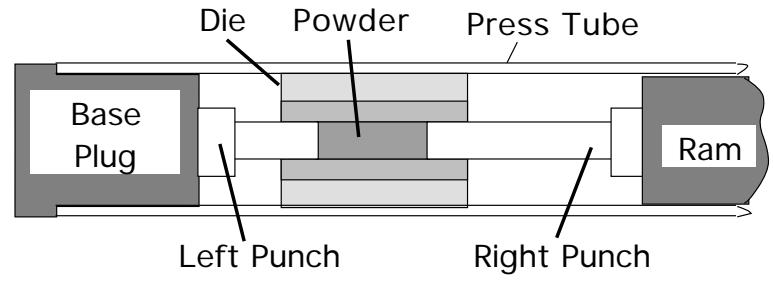

Fig. 2(b). Press tube interior 
magnetic field of the SCM and forces on the powder and compacts. The length of the press tube was chosen to enable access to the SCM centered $38-\mathrm{cm}$ within its helium dewar, and to locate the more magnetic hydraulic cylinder in the far field of the SCM, where the field gradient is weak. Also, the press tube could be rotated, which allowed die/punch set insertion in an even weaker field. See Fig. 3. The insertion mechanism and press tube were sized to withstand the magnetic forces and behave essentially as rigid bodies.

For automated factory operation, press operations are envisioned to occur from both ends of the SCM bore, where the extraction of one press tube is synchronized with the insertion of the other. Powder filling of one die would occur while another compact is being pressed. The design of both presses and the transport mechanism would minimize magnetic forces during extraction and insertion, by selective location of the powder, compacts and magnetic shimming at opposite ends of the SCM. Magnetic shielding of the SCM would allow powder filling of the die and compact ejection, when that press is in the extracted position.

\section{B.Superconducting Solenoid (SCM)}

A Cryomagnetics, Inc. SCM was purchased, which has a horizontal, 7.6-cm diameter warm-bore and is similar to other designs they have fabricated. The length of the bore tube is $76.2 \mathrm{~cm}$. The magnitude of the steady field in the bore of the SCM can be continuously varied up to 9 Tesla. PM powder can be aligned in a field that is uniform within $5 \%$, over a volume that is large enough to press $2.54-\mathrm{cm}$ diameter cylindrical PMs that are over 10-cm long. SCM cooling was achieved with only liquid helium and super-insulation. A cryo-cooler and magnetic shielding for the SCM were not included, because these additions would have more than doubled the initial cost. Both additions will be needed for acceptance into factory operations. In retrospect, the SCM should have been shielded for laboratory use. The laboratory was large enough to isolate the far field and satisfy all environmental, health, and safety issues, but the cost of shielding nearby computer monitors, which are sensitive to $\mathrm{mT}$, nearly equaled the cost of initially shielding the SCM.

The robustness of the mechanical components holding the

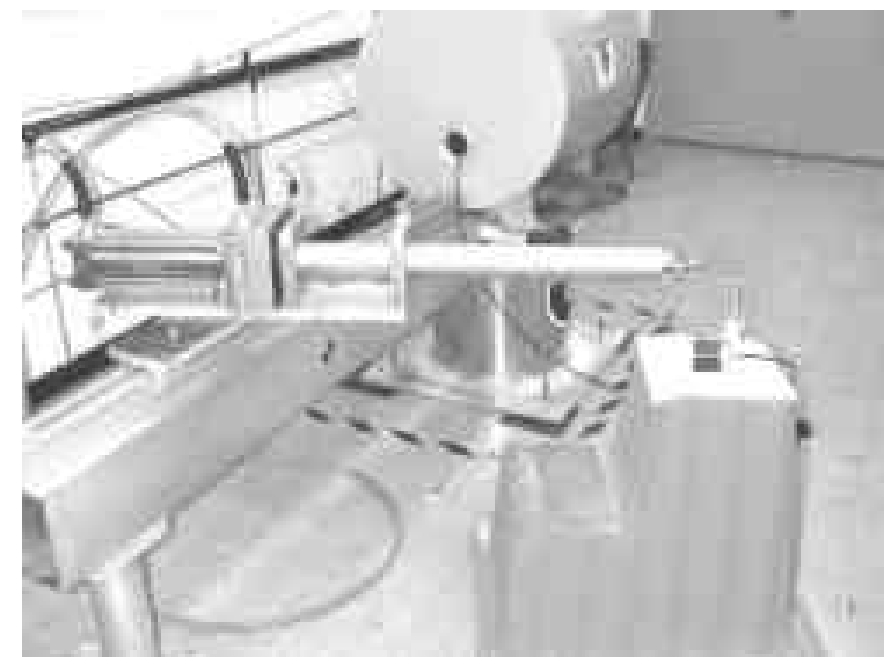

Fig. 3. Die/punch insertion (right) into press tube, rotated into loading position. Hydraulic cylinder on back (left) end of press tube.
$\mathrm{SCM}$, in its liquid helium dewar, were specifically designed to allow for insertion and extraction of the magnetic powder and compacts, while operating at $9 \mathrm{~T}$. Calculations showed that forces along the magnet axis could be as large as $3402 \mathrm{~N}$ (750 lbs.). Prior to acceptance of the SCM, the magnetic field in the bore was verified at the manufacturer, as well as the SCM support structure. A piece of steel was inserted into and extracted from the SCM operating at $9 \mathrm{~T}$, which created the maximum magnetic forces expected.

The 42,000-turn SCM was 40-cm long and had inner and outer diameters of $12.75 \mathrm{~cm}$ and $20.1 \mathrm{~cm}$, respectively. Each end had an additional 2800-turn, 7.9-cm long shim coil. Variations in the diameter of the $\mathrm{Nb}-\mathrm{Ti} / \mathrm{Cu}$ wire in the main coil and the shim coils were used to achieve the magnetic field uniformity desired. A 75-A powder supply was more than adequate to operate the SCM at 9-T. For reasons of laboratory safety only, the SCM was operated in the power mode, rather than the persistent mode. Between uses, the SCM was de-energized. Ramp times were about 0.2$\mathrm{T} /$ minute. Solenoids with much faster rates are available at additional cost. In retrospect, heating coils should have been included, to intentionally quench the solenoid. After several cycles, remnant fields of $5 \mathrm{mT}$ to $10 \mathrm{mT}$, with sharp axial gradients, were present at each end of the SCM. The super currents circulating around the diameters of the wires that were responsible for remnant fields can only be removed by quenching. When operating at high fields, the remnant fields were not a concern. But part of the testing included the removal of loose magnet powder from the de-energized SCM bore. Custom means of shielding these specimens had to be designed and used.

\section{Die and Punch Set}

The 8-mm axial-die and punch set shown in Fig. 4 was designed in consultation with the permanent-magnet manufacturer Magnequench UG, Inc. and Bronson and Bratton, Inc., a tooling fabricator. The set was made using very low magnetic-permeability material $(<1.002)$, which will not effect significantly the uniformity of the SCMs magnetic field nor the alignment of the magnet powder. Still, when operating at 8-T, a force of $136 \mathrm{~N}$ was required

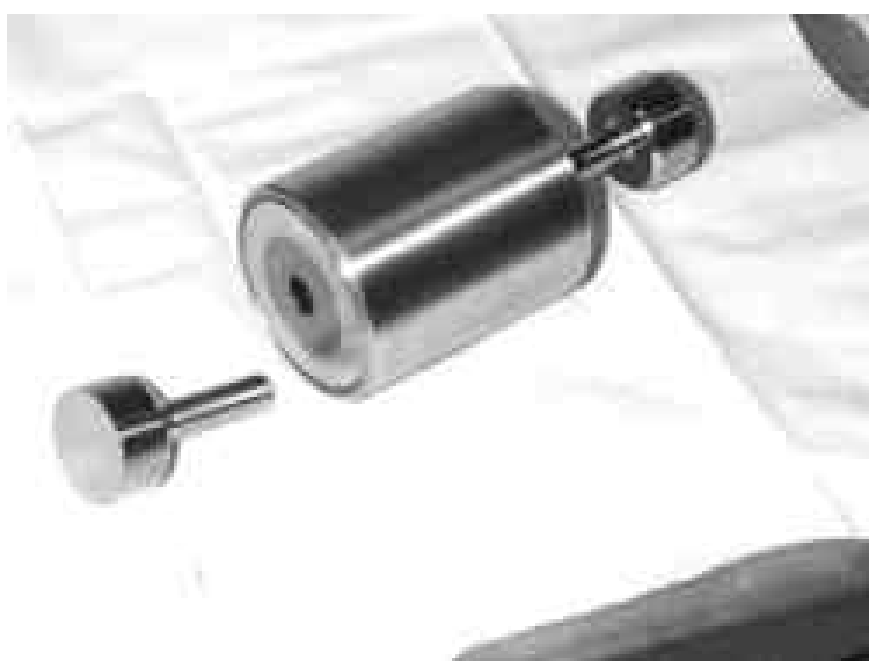

Fig. 4. One punch removed from die. 
to extract the empty die/punch set from the SCM. The die insert and the punches were made of nickel carbide, while the die case was made of Inconel 718. Tight diametrical clearances $(<0.025 \mathrm{~mm})$ were required to maintain alignment of this simple die and punch set. The tooling was polished to mirror finishes and very-light coatings of stearic acid lubricants were used to insure "floating die" operation. Often, industry uses tool steel with carbide inserts and tips, but they must magnetically shim the electromagnet, for each magnet geometry, to achieve a uniform alignment field. Clearly, tool steel would be difficult to use in an SCM's reciprocating press.

\section{Permanent Magnet Results}

Sintered NdFeB PM production is a mature industry that was begun in the middle 1980s. However, they continue to provide the highest energy product PMs available. Today's PM's magnetic properties are within $5-25 \%$ of their theoretical maximums. Industry considers an improvement in properties of $2 \%$ to $3 \%$ as significant. Magnets that are axialdie pressed and sintered to near-final-shape have magnetic properties farthest from their theoretical maximums, but they are the lowest in cost to make. A major goal of industry is to make better near-final-shape PMs by axial-die pressing.

More than $250 \mathrm{NdFeB}$ cylindrical compacts have been pressed in the ANL facility, using production-grade magnet powder obtained from the industrial partner, Magnequench UG. Subsequently, many batches of the anisotropic compacts, with their grain orientation mechanically locked in place, were returned to our partner for sintering, annealing, machining, and the measurement of engineering magnetic properties. The first PMs had properties that were far from optimal, thus a significant effort was made in cooperation with our partner to optimize processing at Argonne.

Compacts with a length/diameter $\mathrm{L} / \mathrm{D} \sim 1$ were made as part of a study to correlate energy-product improvements with increases in the alignment. In this early study, most of the compacts were made with the alignment fields applied only just before and during the pressing. Results are given in Fig. 5. The maximum energy product BHmax was increased by $\sim 12 \%$ by tripling the 2 -T maximum alignment field available with electromagnets. Unexpectedly, the best magnets (solid symbols) were made when the SCM and the alignment field was on for all operations. This condition simulates the severe field gradients that loose powder in the die would experience during insertion into an operating superconducting solenoid by a reciprocating press. Of most importance, these maximum energy products are comparable to those of more expensive magnets made by other pressing techniques. About $92 \%$ of the theoretical maximum was achieved.

A study of near-final-shape cylindrical magnets has been completed $[2,4]$ for compacts with three different length-todiameter ratios, $\mathrm{L} / \mathrm{D}=0.25,0.50$, and 0.73 . The remnant magnetization, $\mathrm{Br}$, of the shortest magnets, improved the most. Quadrupling the alignment field from $2 \mathrm{~T}$ increased $\mathrm{Br}$ by greater than $7 \%$. This is equivalent to a $15 \%$ increase in the maximum energy product, since $\mathrm{BHmax}$ is $\sim \mathrm{Br}^{2}$. Even larger improvements in energy product are expected, when shorter PMs are made.

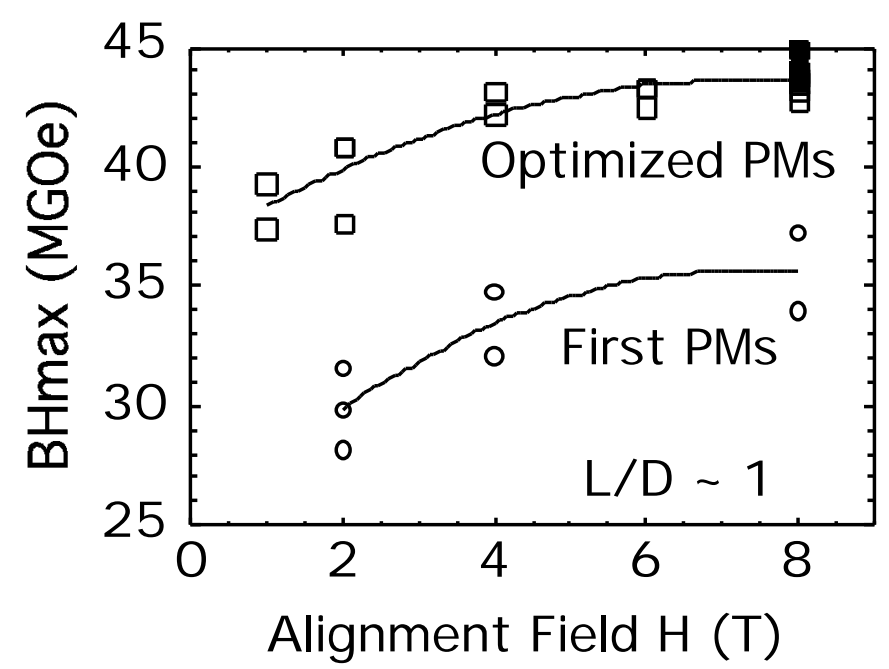

Fig. 5. Energy product, BHmax, was increased significantly by applying alignment fields higher than those possible with industry's electromagnets.

\section{CONCLUSIONS}

The maximum energy products of axial-die pressed PMs were improved significantly by the use of SCMs, to the quality of the more expensive magnets obtained by transverse-die pressing. This, alone, represents an opportunity for significant cost savings. The main barrier to using a SCM with a reciprocating press was shown not to be the show stopper that industry expected. In fact, the best magnets were made when batch processing simulated the conditions of the reciprocating feed. The energy products were within $92 \%$ of their theoretical maximum. Apparently, insertion of loose powder into the strong field gradients of the SCM provides a form of magnetic die filling that improves grain alignment.

\section{REFERENCES}

[1] I. R. Harris, "Magnet processing," in Rare-Earth Iron Permanent Magnets, Ed. J.M. D. Coey Oxford: Clarendon Press, 1996, pp. 336380.

[2] T. M. Mulcahy, E. Rozendaal, J. H. Wise, and J. R. Hull, "NdFeB Magnets Aligned in a 9-T Superconducting Solenoid," Seventeenth International Workshop on Rare Earth Magnets and Their Applications, August 18-22, 2002, submitted for publication.

[3] J. Boehm, "Magnetic Separation-Superconductivity in Industry," IEEE Trans. Applied Superconductivity, vol. 10(1), March 2000, pp. 710-715.

[4] T. M. Mulcahy, E. Rozendaal, J. H. Wise, J. R. Hull, and L. R Turner, "Improving Sintered NdFeB Permanent Magnets by Powder Compaction in a 9-T Superconducting Solenoid," 47-th Magnetism and Magnetic Materials Conference, November 2002, submitted for publication. 